The ASTROPHYSICAL Journal, 251:417-423, 1981 December 15

(c) 1981. The American Astronomical Society. All rights reserved. Printed in U.S.A.

\title{
TOWARD THE APPLICATION OF A METRIC SIZE FUNCTION IN GALACTIC EVOLUTION AND COSMOLOGY
}

\author{
S. DJORGOVSKI AND H. SPINRAD ${ }^{1}$ \\ Astronomy Department, University of California, Berkeley \\ Received 1981 March 12; accepted 1981 June 23
}

\begin{abstract}
We have measured the metric size function $\eta(r)$ defined by Petrosian on a limited sample of luminous distant galaxies, up to a redshift of 1.175. Although our investigation has a preliminary character, we find some evidence for the evolution of elliptical galaxies. Using the Ostriker-Hausman theoretical models, we place some constraints on the rates of dynamical evolution.

The eta function appears to be an extremely sensitive probe for the investigation of galactic evolution and potentially useful in cosmology. Some problems and directions for future work are outlined.
\end{abstract}

Subject headings: cosmology — galaxies: evolution — galaxies: photometry

\section{INTRODUCTION}

The eta function was introduced first by V. Petrosian (1976), as a tool for the investigations of brightness evolution of galaxies, independent of cosmology and redshift. (See also Tinsley 1976). It is defined as:

$$
\eta(r)=2 \frac{d(\log r)}{d\left[\log L_{\nu}(r)\right]},
$$

where $r$ is the radius at which quantities are determined, and $L_{\nu}(r)$ is the total luminosity inside of $r$. Neglecting the frequency dependence, it is easy to see that eta actually represents the ratio of the surface brightness averaged within radius $r$, to the local surface brightness at $r$ itself:

$$
\eta(r)=\frac{\langle S B\rangle_{r}}{S B_{r}} .
$$

We prefer to use the logarithmic expression, changing sign for convenience:

$$
\eta(r)=\eta_{r}=\mu_{r}-\langle\mu\rangle_{r},
$$

where all quantities are now in magnitudes per square arcsec. We have measured radii at a level at which this function reaches $2 \mathrm{mag} \operatorname{arcsec}^{-2}$, i.e., where the surface brightness is approximately one-sixth of the average within the diaphragm of that radius. This provided, in our opinion, a good compromise between the distortion due to seeing at the smaller radii and the sky background noise at the larger.

\footnotetext{
${ }^{1}$ Visiting astronomer, Kitt Peak and Cerro Tololo Observatories, operated by the Association of Universities for Research in Astronomy, under the contract with the National Science Foundation.
}

Since the relation between the observed and the rest frame bolometric surface brightness is a function of the redshift alone in all relativistic models:

$$
S B_{\mathrm{rf}}=S B_{\mathrm{obs}}(1+z)^{4},
$$

it is obvious that $\eta$ will not depend on redshift, providing that the metric radial scale is the same. Because no assumptions were made about the cosmology or form of the galactic light profile itself, eta represents the modelindependent, metric radial scale for the given galaxy. This direct metric character is of great advantage, as compared to the isophotal determinations of the radius, which require knowledge about the profile itself, in order to be converted to a physically meaningful local metric scale. Thus, eta can be employed to define a standard diaphragm for galactic photometry, avoiding the need for the usual iterative aperture correction procedure. On the other hand, the shape of the galactic profile can be investigated, since it is possible to plot the rest frame surface brightness versus eta directly. We have also attempted to apply an other metric scale on the same data, namely the fixed fraction of the light radius (e.g., the de Vaucouleurs "effective radius"). Our experiments show that for the faint objects we are dealing with, this scale suffers very much from imprecise sky subtraction.

The relation between the eta scale and the galaxian radius in physical units is illustrated in Figure 1 for the relatively bright galaxy No. 1 in the Abell 520 cluster.

Eta can be also related to the Gunn-Oke structural parameter alpha:

$$
\eta=-2.5 \log \left(\frac{\alpha}{2}\right)
$$




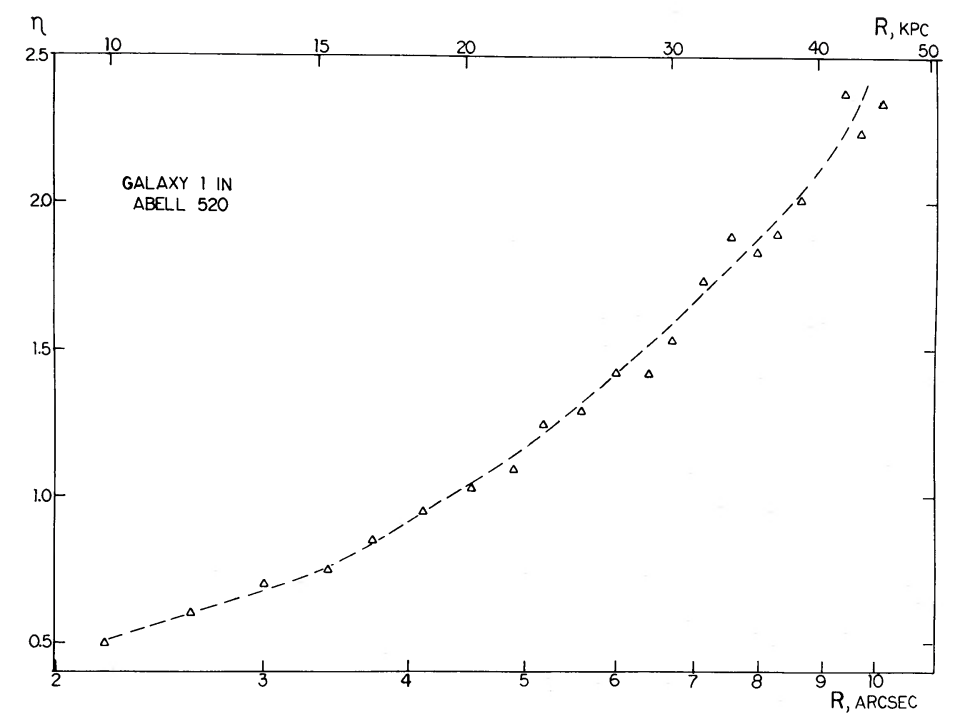

FIG. 1.- The relation between the eta radial scale in units of mag $\operatorname{arcsec}^{-2}$, and radius in physical units of $h_{50}{ }^{-1} \mathrm{kpc}$, illustrated for the example of galaxy No. 1 in the Abell 520 cluster. The broken line is a visual estimate of the profile.

(Petrosian 1976; Ostriker 1980). This connection will be exploited for the comparison with the theoretical dynamical evolutionary models. Note, however, that this definition does not specify in advance the physical size of the radius, which is generally unknown, and which is dependent on cosmology. Since $\eta$ is observable, defined in this way, $\alpha$ is also an observable quantity.

\section{OBSERVATIONS AND DATA REDUCTION}

We have used the first- and second-ranked ellipticals or $\mathrm{cD}$ galaxies in rich clusters and, at the higher redshifts, strong radio emitters, which are presumably either of the above (Table 1). It is possible, though, that some of the very distant objects are spirals, their structures being indistinguishable due to the seeing. However, all at $z>0.55$ except the galaxy No. 1 in cluster $1305+2952$ are strong radio emitters, and therefore most likely ellipticals or cD's. This procedure for choosing the "standard candle" is certainly imperfect, and the sample is neither statistically complete nor homogeneous, but we desire to utilize all the high-redshift data. Therefore, although our photometric data are few (only 25 galaxies), they reach up to redshift of 1.175 , thus providing us with a glimpse of changes involved over very long time scales.

Table 1 shows the properties of the galaxies used in our investigation. The radius is measured at a level at which $\eta=2 \mathrm{mag} \operatorname{arcsec}^{-1}$ and is expressed in arcsec. The weight (col. [7]) is a statistical weight determined subjectively by the quality of available data. Column (9) gives the rest frame surface brightness at the $\eta=2$ radius, and column (10) is the apparent red magnitude within the $\eta=2$ diaphragm.
The raw photometric material consists of calibrated (mostly IIIa-F) $4 \mathrm{~m}$ prime focus plates, taken at the Kitt Peak and Cerro Tololo Observatories. The plates were traced with the Berkeley PDS microdensitometer and reduced with an assumed sky brightness of $\mu_{\text {sky }}=21.5$. The two-dimensional editing techniques follow Mathieu and Spinrad (1981) and Spinrad, Stauffer, and Harlan (1979), using the program developed by R. Kron. Some of the direct observed profiles are shown in Figure 2. Our experiments with PDS surface photometry show that if the sky is not badly contaminated by other galaxies, subtraction can be done with satisfactorily precise reproducibility. A major problem, however, is the "pollution" of images by other cluster members. Their images are smoothed by seeing, and they may be themselves undetectable. It is very difficult to do satisfactory subtraction in such cases. Save for this smoothing, the seeing point spread function apparently does not matter very much at the large radii we are using; it would be crucial, however, for any investigation of the core regions.

Since all of our objects are on different plates, and since most of the data for nearby $\mathrm{E}$ galaxies have been produced by others, we could not establish internally consistent photometry with the desirable zero point precision (say, $0.1 \mu$ ), but rather with a dispersion of the order of $0.6 \mu$ (as inferred from the $\mu_{\mathrm{rf}} / z$ plot). This, of course, does not matter with the eta function (the difference in shifts cancels exactly), but it makes it difficult to draw quantitative conclusions about the changes in overall brightness of galaxies as a function of redshift, or for internal comparisons. For this reason we do not employ the $m$ versus $z$ test in our analysis. We did, 
TABLE 1

THE GALAXIES USED IN OUR INVESTIGATION

\begin{tabular}{|c|c|c|c|c|c|c|c|c|c|c|}
\hline $\begin{array}{l}\text { No. } \\
(1)\end{array}$ & $\begin{array}{c}\text { Galaxy/Cluster } \\
\text { (2) }\end{array}$ & $\begin{array}{c}z \\
(3)\end{array}$ & $\begin{array}{c}r \\
(\operatorname{arcsec}) \\
(4)\end{array}$ & $\begin{array}{c}\log (r) \\
(5)\end{array}$ & $\begin{array}{l}\Delta \log (r) \\
\quad(6)\end{array}$ & $\begin{array}{l}\text { Weight } \\
\text { (7) }\end{array}$ & $\begin{array}{l}\text { Type } \\
(8)\end{array}$ & $\begin{array}{l}\mu_{\mathrm{rf}} \\
(9)\end{array}$ & $\begin{array}{c}m \\
(10)\end{array}$ & $\begin{array}{l}\text { Reference } \\
\text { (11) }\end{array}$ \\
\hline $1 \ldots$ & N4472/Virgo & 0.00317 & 245 & 2.39 & 0.027 & $1 / 2$ & $\mathrm{E}$ & 24.6 & 9.36 & 1 \\
\hline $2 \ldots$ & N1316/For A & 0.00545 & 237 & 2.375 & 0.028 & $1 / 2$ & $\mathrm{cD}$ & 24.5 & 9.41 & 2 \\
\hline $3 \ldots$ & N3862/A1367 & 0.0218 & 128 & 2.107 & 0.054 & $1 / 2$ & E & 24.8 & 11.08 & 3 \\
\hline $4 \ldots$ & N6158/A2199 & 0.0312 & 40 & 1.60 & 0.058 & 1 & E & 24.1 & 12.86 & 4 \\
\hline $5 \ldots$ & No. 1 in $\mathrm{A} 1228$ & 0.0344 & 49 & 1.69 & 0.027 & $1 / 2$ & E & 24.4 & 12.67 & 4 \\
\hline $6 \ldots$ & N6041/A2151 & 0.036 & 54 & 1.73 & 0.025 & $1 / 2$ & S0-E & 24.4 & 12.47 & 4 \\
\hline $7 \ldots$ & No. 2 in A401 & 0.0746 & 16.4 & 1.215 & 0.088 & 1 & $\mathrm{E}$ & 24.0 & 14.69 & 5 \\
\hline $8 \ldots$ & No. 2 in $A 2670$ & 0.0753 & 19.5 & 1.29 & 0.073 & 1 & $\mathrm{E}$ & 24.3 & 14.60 & 5 \\
\hline $9 \ldots$ & No. 1 in A795 & 0.140 & 13.34 & 1.125 & 0.017 & 1 & $\mathrm{E}$ & 24.8 & 16.50 & 6 \\
\hline $10 \ldots$ & $\begin{array}{l}\text { No. } 1 \text { in Hydra II } \\
\text { cluster }\end{array}$ & 0.201 & 5.45 & 0.736 & 0.042 & 1 & $\mathrm{E}$ & 23.3 & 17.19 & 6 \\
\hline $11 \ldots$ & No. 1 in $\mathrm{A} 520$ & 0.203 & 8.51 & 0.93 & 0.026 & 1 & E & 24.3 & 17.17 & 6 \\
\hline $12 \ldots$ & $\begin{array}{l}\text { No. } 1 \text { in cluster } \\
\text { Zw } 1305+2941\end{array}$ & 0.240 & 10.72 & 1.03 & 0.021 & 1 & $\mathrm{cD}$ & 24.35 & 17.24 & 6 \\
\hline $13 \ldots$ & $\begin{array}{l}\text { No. } 1 \text { in cluster } \\
\text { Corwin } 0404\end{array}$ & 0.300 & 8.28 & 0.918 & 0.027 & 1 & $\mathrm{E}$ & 24.15 & 17.44 & 6 \\
\hline $14 \ldots$ & $\begin{array}{l}\text { No. } 1 \text { in cluster } \\
0237-0138\end{array}$ & 0.373 & 8.32 & 0.92 & 0.027 & 1 & $\mathrm{E}$ & 24.6 & 18.29 & 6 \\
\hline $15 \ldots$ & $\begin{array}{l}\text { No. } 1 \text { in cluster } \\
0949+4409\end{array}$ & 0.385 & 3.55 & 0.55 & 0.066 & 1 & $\mathrm{E}$ & 23.8 & 19.17 & 6 \\
\hline $16 \ldots$ & $\begin{array}{l}\text { No. } 1 \text { in cluster } \\
\quad 0024+16\end{array}$ & 0.392 & 5.31 & 0.725 & 0.043 & 1 & $\mathrm{E}$ & 24.9 & 19.50 & 6 \\
\hline $17 \ldots$ & $3 C 295$ & 0.461 & 6.17 & 0.79 & 0.037 & 1 & $\mathrm{cD}:$ & 24.0 & 18.43 & 7 \\
\hline $18 \ldots$ & PKS 0400-64 & 0.480 & 4.68 & 0.67 & 0.049 & 1 & $\mathrm{E}$ & 24.1 & 19.24 & 8 \\
\hline $19 \ldots$ & $\begin{array}{l}\text { No. } 1 \text { in cluster } \\
0016+16\end{array}$ & 0.541 & 5.01 & 0.70 & 0.032 & $1 / 2$ & $\mathrm{E}$ & 25.0 & 20.2 & 6 \\
\hline $20 \ldots$ & $\begin{array}{l}\text { No. } 2 \text { in } \\
\text { 3C } 330 \text { cluster }\end{array}$ & 0.545 & 4.37 & 0.64 & 0.053 & 1 & $\mathrm{E}$ & 24.0 & 20.23 & 9 \\
\hline $21 \ldots$ & PKS $0116+082$ & 0.593 & 4.22 & 0.625 & 0.055 & 1 & $\mathbf{R}$ & 24.7 & 20.48 & 9 \\
\hline $22 \ldots$ & $3 \mathrm{C} 352$ & 0.806 & 3.48 & 0.542 & 0.067 & 1 & $\mathrm{R}$ & 24.6 & 21.27 & 10 \\
\hline $23 \ldots$ & $\begin{array}{l}\text { No. } 1 \text { in cluster } \\
1305+2952\end{array}$ & 0.942 & 3.02 & 0.48 & 0.079 & 1 & cD: & 23.3 & 20.18 & 11 \\
\hline $24 \ldots$ & $3 \mathrm{C} 13$ & 1.05 & 2.2 & 0.343 & 0.041 & $1 / 2$ & $\mathrm{R}$ & 22.7 & 20.75 & 12,13 \\
\hline $25 \ldots$. & 3C 427.1 & 1.175 & 2.0 & 0.30 & 0.046 & $1 / 2$ & $\mathbf{R}$ & 23.9 & 22.0 & 12,13 \\
\hline
\end{tabular}

ReFERENCES.-(1) King 1978. (2) Schweizer 1980. (3) S. E. Strom and K. M. Strom 1978. (4) K. M. Strom and S. E. Strom 1978. (5) Strom and Strom 1979. (6) Spinrad unpublished. (7) Mathieu and Spinrad 1981. (8) Spinrad, Kron, and Hunstead 1979. (9) Spinrad et al. 1976. (10) Smith et al. 1979. (11) Kron, Spinrad, and King 1977. (12) Spinrad, Stauffer, and Butcher 1981. (13) Smith, Burbidge, and Spinrad 1976.

however, use the Hubble diagram as a crude external check for our qualitative conclusions.

\section{COSMOLOGICAL TEST WITH $\eta$ : A SIGNATURE OF THE EVOLUTION}

As noted by Hickson and Adams (1979a), the existing cluster size data may provide information on both cosmology and galactic evolution. In order to use them fully, one must not make any a priori assumptions about the cosmological models before the data reduction. Following this principle, we have calculated our statistics in a wide range of Friedman-Robertson-Walker models, including the physically meaningless ones. The appropriate formulas can be found in Refsdal, Stabell, and De Lange (1967) or Hickson and Adams (1979a), and we will not repeat them here.
To perform the cosmological test using the $\eta$ determined radii, one must make the following assumptions: (1) the shape of the light profile is the same for all objects used; (2) the characteristic radius is the same; and (3) the radial color gradients do not change with the redshift. Giant E galaxies may well satisfy these assumptions.

In order to obtain the best fit to our data, viz., $\eta$ angular size versus redshift plots for different cosmological models, we minimized the sum of the least squares residuals, weighted with the individual errors $\delta_{i}$ and the weight $w_{i}$, subjectively determined by the quality of the particular data:

$$
\sum_{i=1}^{25}\left(\frac{w_{i}}{\delta_{i}}\right)^{2}\left[\log r_{i}-\log r_{\mathrm{th}}\left(z_{i}, q_{0}, \sigma_{0}, R\right)\right]^{2},
$$




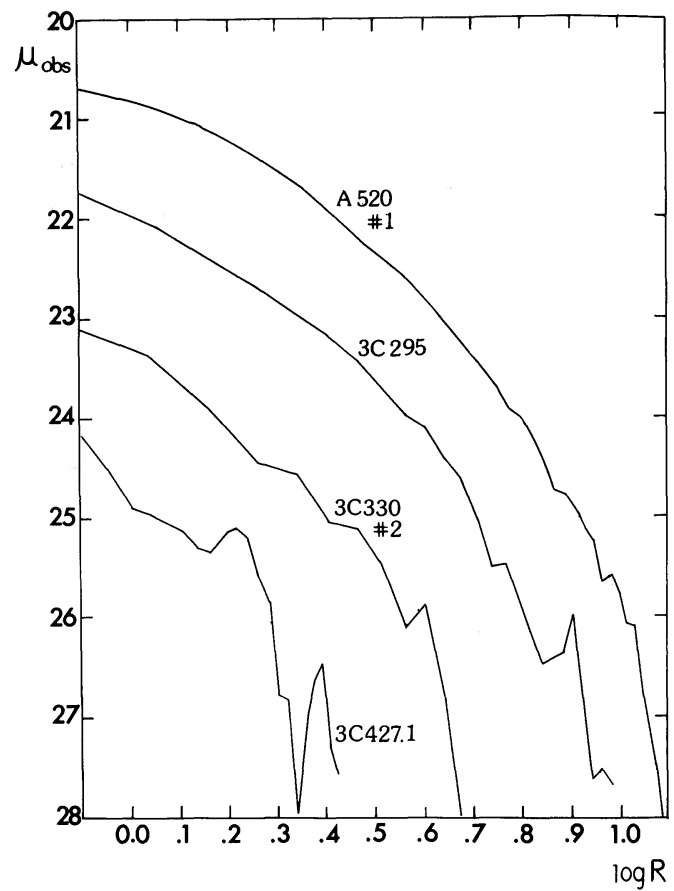

FIG. 2.-Some examples of the observed $S B$ profiles ( $R$ unit is arcsec): 3C 427.1 is the faintest and most distant object in our sample; 3C 295 is the worst case of "pollution"; \#2 in 3C 330 is faint but a "fair" profile; \#1 in A520 is the galaxy from Fig. 1, given here for comparison.

where $r_{\mathrm{th}}\left(z_{i}, q_{0}, \sigma_{0}, R\right)$ is the theoretical value of the angular radius for the cosmological model assumed, $R$ is the value of the galactic radius in physical units $(\mathrm{kpc})$, and

$$
\delta_{i}=\Delta \log r_{i}=\log \left(\frac{r_{i}}{r_{i}-\Delta r_{i}}\right)
$$

is adopted as a conservative error for the $\log r_{i}$. This does not include the intrinsic dispersion of $\log r$ completely; to determine it, one would need a much larger sample than ours.

Also, we minimized the sum of nonweighted leastsquares residuals:

$$
\sum_{i=1}^{25}\left[\log r_{i}-\log r_{\mathrm{th}}\left(z_{i}, q_{0}, \sigma_{0}, R\right)\right]^{2}
$$

and obtained very similar results.

We used the logarithms of radii rather than radii themselves because it seems more plausible to assume that logarithms rather than radii are distributed normally around some mean value. Although in reality the error in radius is a combination of both linear and logarithmic errors, our experiments show that the logarithmic part is fairly dominant. This provides some justification for the application of a least-squares crite- rion as described above. We did try some other statistical approaches, including calculations with the radii in linear form (which has a tendency to diverge), but neither of those attempts proved to be more statistically justifiable.

The above calculations were done over the wide range of free parameters $q_{0}, \sigma_{0}$, and $R$, in search of the global minima. Our best fit to the data is given by the following parameters:

$$
\begin{aligned}
q_{0} & =-0.05_{-1.1}^{+1.6} \\
\sigma_{0} & =-0.3_{-1.1}^{+1.6} \quad(1 \text { sigma errors }) \\
\langle R\rangle & =\left(46_{-9}^{+2}\right) h_{50}^{-1} \mathrm{kpc} .
\end{aligned}
$$

This result is shown in Figure 3 (solid line).

The probability contours in the plane of best radius are shown in Figure 4. The negative values of the density parameter $\sigma_{0}$ are slightly preferred. These have no physical meaning in any relativistic model, with or without a cosmological constant. Therefore, we have a signature of unanticipated systematic effects.

Elongated features of the probability ellipsoids appear in all of our statistics, and can be also noted in Hickson and Adams (1979b). They are apparently caused by the degeneracy in the $\left\{q_{0}, \sigma_{0} \mid z\right\}$ planes, namely the fact that isodistant lines (i.e., where the proper frame distance $\omega=$ const.) are quasi-parallel to the line of zero curvature, $3 \sigma_{0}-q_{0}-1=0$.

Although our data do not favor strongly any particular cosmological model and certainly do not satisfy standards of homogeneity and completeness as would be desired in cosmological investigations, we think that models with cosmological constant $\Lambda \neq 0$ should not be a priori discarded. There is growing observational evidence that $\Lambda$ may have a nonzero value (see, e.g., Tinsley 1978), and there are also some theoretical indications coming from quantum field theory that some energy density may be associated with the deformations of the physical vacuum, viz., the expansion of the universe (Kolb and Wolfram 1980; Canuto and Lee 1977; Hickson and Adams 1979 $a$ and references therein). This is a question which remains to be settled by future observational and theoretical developments. But at least future data reduction should be done without the usual arbitrary assumption that $\Lambda=0$.

\section{THE INFLUENCE OF DYNAMICAL EVOLUTION}

After the best fit was obtained, we looked for possible systematic effects which might have changed the form of the $\log r$ versus $\log z$ lines, like those in Figure 3. We have looked into a wide range of possible influences, and there are several which seem to be important.

Most readily understandable are the selection effects: one might tend to pick the brighter and bigger objects at 


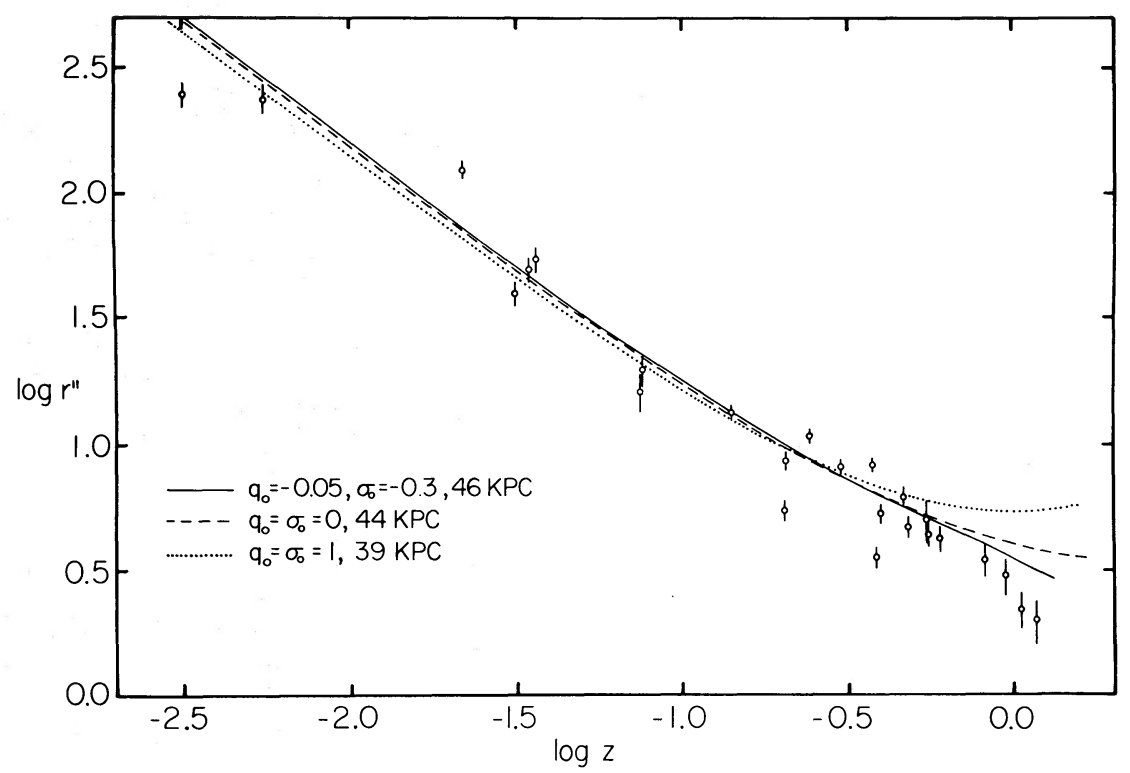

FIG. 3.-A test of cosmological metric size vs. redshift. The best fit to our data is represented by the solid line. Two standard Friedman models are shown for comparison.

the high redshifts, thus violating the assumption of the constant radial scale. Also, the fact that many of our galaxies were initially discovered because of their strong radio emission may produce another bias (the possibility of optically active nuclei). Unfortunately, because our sample is far from being statistically complete, we are not able to put forward any quantitative estimate of this effect or to introduce suitable corrections.

Another potential problem is brightness evolution of the galaxies, due to post-main-sequence evolution and possibly the changes in the star formation rate (see, e.g., Tinsley and Gunn 1976). However, the $\eta$ test is affected

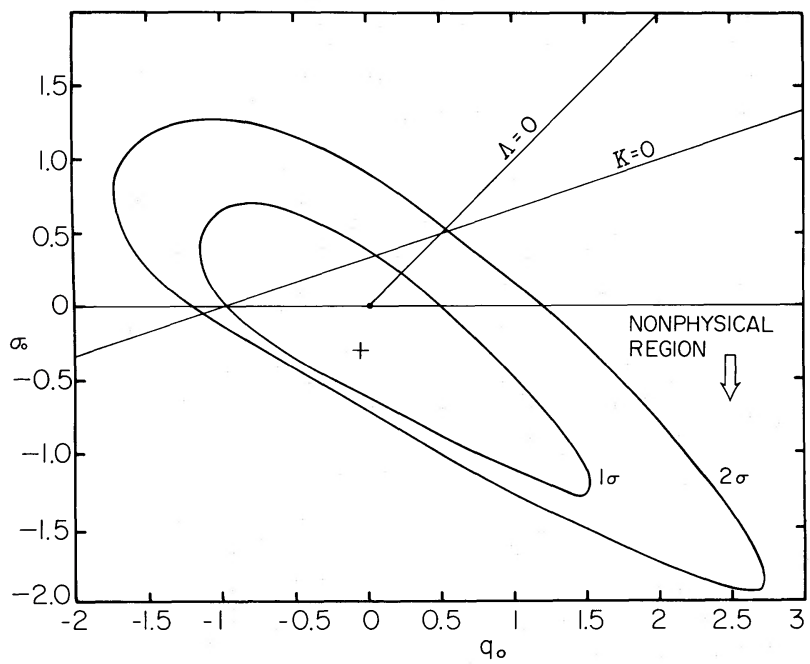

Fig. 4. - A cut through the probability ellipsoids in the $\left(q_{0}, \sigma_{0}\right)$ plane of the "best" radius. The best fit is marked with the cross. only if the shape of the light profile and radial color gradients change.

Both of these effects almost undoubtedly exist at some level, and both will probably systematically tend to bend our $\log r / \log z$ lines. There does exist, however, an important effect to decrease the galaxian size with increasing redshift, namely the dynamical evolution of galaxies, due to cannibalism (Ostriker and Hausman 1977). These authors have calculated the evolution of the parameter $\alpha$ at $16 h_{50}{ }^{-1} \mathrm{kpc}$, as a central big galaxy gobbles its smaller neighbors (Hausman and Ostriker 1978). Galaxies then evolve in a sense that they are seen as smaller at the higher redshifts, thus effectively bending the $\log r / \log z$ lines downward and lowering the observed values of $q_{0}$ and $\sigma_{0}$. This model accounts for the redistribution of luminous matter, but it does not treat increased stellar formation satisfactorily. Although the size of the dynamical effect is still controversial (Gunn and Tinsley 1976), it is undoubtedly very important for central $\mathrm{cD}$ and $\mathrm{E}$ systems.

The time scales involved are dependent on the galaxy and the cluster parameters, which are neither uniform nor easily determinable in our sample. We calculated some possible effects using a wide range of the relevant parameters with the Ostriker-Hausman estimates for the rates of evolution of $\alpha$. Our best estimates resulted in very high values of $q_{0}$ (up to 10 or more). We conclude then that this may be some indication that in reality several opposing phenomena take place, in which dynamical evolution barely dominates over the possible effects of stellar evolution gradients (governed by a galaxy's own "nuclear clock") and the selection effects, if these are important in the above mentioned sense. 

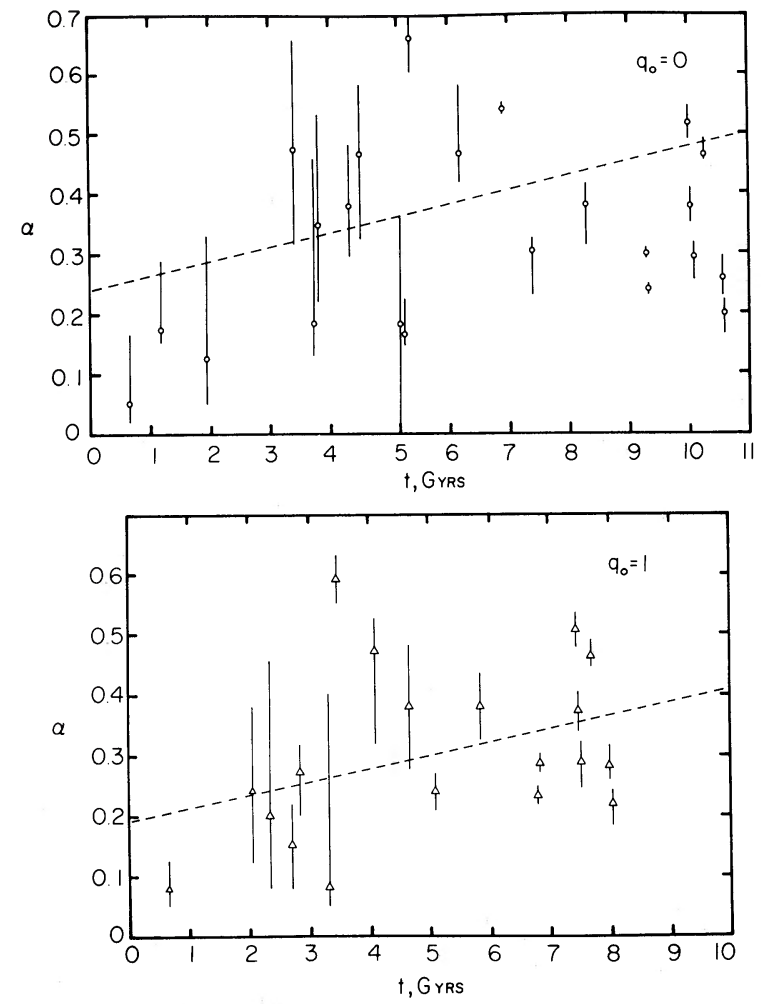

Fig. 5.- (Top): Evolution of the structure parameter $\alpha$ at the radius of $40 h_{50}^{-1} \mathrm{kpc}$, as inferred from our data, assuming the simple cosmology with $q_{0}=0, H_{0}=50 \mathrm{~km} \mathrm{~s}^{-1} \mathrm{Mpc}$. Time zero is set arbitrarily at $z=1.2$. The broken line is the least squares fit, not weighted with the error bars. (Bottom): Same as (top), but for $q_{0}=1$.

Cannibalism must dominate, however, since our best fits give physically meaningless negative values of the density parameter $\sigma_{0}$. This is in agreement with the Hickson and Adams's (1979b) result.

In order to find the rates of dynamical evolution needed to produce changes from a "plausible" cosmology to the "observed" values of $q_{0}$ and $\sigma_{0}$, we then did the opposite calculation, determining the effective change of alpha, by assuming the simple Friedman models with $q_{0}=\sigma_{0}=0,1 / 2$, or 1 , and using our $\eta$ profiles. Of course, since $q_{0}$ and $\sigma_{0}$ are not really known, this investigation had as its purpose only to determine the typical size of the effect needed to produce observed shape of the $\log r / \log z$ best fit line. We have calculated alpha at the radius of $40 h_{50}^{-1} \mathrm{kpc}$, because that is closer to our best fit value of radius and because our data suffer from seeing noise and insufficient resolution at the $16 h_{50}{ }^{-1}$ $\mathrm{kpc}$. It is true that changes of alpha at 16 and 40 (or 46) kpc may differ significantly. Nevertheless, our calculations at the $20 h_{50}{ }^{-1} \mathrm{kpc}$, although much noisier, gave similar results. If one assumes a linear approximation for the $\alpha /$ time relation, the resulting slopes for the $q_{0}=0$ and $q_{0}=1$ cases (as shown in Fig. 5) are
$0.014 h_{50} \mathrm{Gyr}^{-1}$ and $0.022 h_{50} \mathrm{Gyr}^{-1}$, respectively. This is roughly in agreement with the rates of the evolution one may infer from the Ostriker-Hausman models, if one assumes that mass-to-light ratio for the cluster is of the order of magnitude higher than the ratio for the galaxy cannibal. We are somewhat reluctant to assume such a high contrast in mass-to-light ratios; data will then require much less evolution in order to fit assumed values of the cosmological parameters. Still, since all the assumptions involved are subject to large uncertainties, these figures should be taken only as an approximate values. Of course, the linear approximation is not necessarily the best one; as a cluster evolves, alpha should slow down its growth and saturate at some value, when relaxation is complete. Indeed, such a trend seems to exist as one looks at Figure 5, namely that alpha grows faster at the beginning and then scatters around some value, but our data have not enough statistical power to support such a conclusion quantitatively.

\section{SOME PROBLEMS AND FUTURE PROSPECTS}

At every stage of this discussion, the uncertainties are substantial. Our present data do not constitute a very powerful statistical set and are subject to selection effects. Theories of galactic evolution, on the other hand, are not yet quantitatively articulate enough. Our results then should be taken just as qualitative indicators for the mainlines of the underlying picture. But we think that prospects for future quantitative work look promising.

One obvious and demanding need is to enrich our sample, especially in the low redshift region. Properties of the eta function for rich clusters should be fully investigated in relatively nearby ones. This will provide an anchor for our test in a relatively easily investigated region. Some work along these lines has been done by Kron and Jankevics (1981). But, of course, since the high redshift objects are decisive where cosmological and evolutionary models are concerned, each new identification of a distant giant elliptical or cD galaxy will be helpful.

It is also important to investigate further influence of pollution by background and foreground objects, and related to this, the effect of seeing. (Note that the seeing-induced smearing of the profiles will generally make the true $\eta$-radii of the distant galaxies even smaller, thus making the departure from the standard Friedman models even more drastic and therefore evolutionary corrections more important and pronounced.) Because of this, and because the determination of eta is based on differential photometry, applications of eta will be much more fruitful from space observations, e.g., with Space Telescope (Gunn 1979). It may prove interesting to investigate some statistically complete samples, such as $3 \mathrm{CR}$ galaxies, with the eta function. 
One would wish to have another parameter aside from $\alpha$, for comparisons with the theory. Needless to say, a theoretical picture is needed which will treat changes due to both stellar and dynamical evolution in a satisfactory and consistent way. Such a theory should make explicit predictions for the changes in galactic light profiles, expressed through the eta function. For example, the Tinsley and Larson (1979) scheme may be extended and generalized along these lines.

The work of Ellis and Perry (1979) shows that the only way in which galactic photometry can provide enough information for determination (and possibly distinguishing as well) of the cosmological and evolutionary effects, is to use all the available information; namely, not only magnitudes, but also the shapes of the galactic profiles, etc. This work may be regarded as a modest step in this direction.

Let us emphasize again that since eta is so strongly sensitive to the evolutionary effects as compared to cosmology, it is actually a potentially powerful probe for the evolution itself, and in the first approximation one does not have to worry much about cosmology. Thus, one large unknown factor in the whole picture can be temporarily avoided. As our knowledge of galactic evolution improves, questions of cosmology will get another powerful tool.

We are greatly indebted to D. Koo, R. Kron, and I. King for most useful conversations. Much of the initial inspiration for this work is due to Kron and Koo. We thank I. King and F. Schweizer for providing us with unpublished data. S. Hildreth and R. Mathieu helped with the data reduction. H. Spinrad was supported by the National Science Foundation. S. Djorgovski was supported by the Berkeley Astronomy Department. We thank the referee for comments which helped clarify some of the implicit assumptions.

\section{REFERENCES}

Canuto, V., and Lee, J. F. 1977, Phys. Letters, 723, 281.

Ellis, G. F. R., and Perry, J. J. 1979, M.N.R.A.S., 187, 357.

Gunn, J. E. 1979, in IAU Colloquium 54, Scientific Research with the Space Telescope, ed. M. S. Longair and J. W. Warner (Washington: Government Printing Office), p. 192

Gunn, J. E., and Tinsley, B. M. 1976, Ap. J., 210, 1.

Hausman, M. A., and Ostriker, J. P. 1978, Ap. J., 224, 320.

Hickson, P., and Adams, P. J. 1979a, Ap. J. (Letters), 234, L87. $1979 b$, Ap. J. (Letters), 234, L91.

King, I. R. 1978, Ap. J., 222, 1

Kolb, E., and Wolfram, S. 1980, Ap. J., 239, 428.

Kron, R. G., and Jankevics, A. 1981, in preparation.

Kron, R. G., Spinrad, H., and King, I. R. 1977, Ap. J., 217, 951.

Mathieu, R., and Spinrad, H. 1981, Ap. J., in press.

Ostriker, J. P. 1980, private communication.

Ostriker, J. P. and Hausman, M. A. 1977, Ap. J. (Letters), 217, L125.

Petrosian, V. 1976, Ap. J. (Letters), 209, L1.

Refsdal, S., Stabell, R., and De Lange, F. G. 1967, Mem. R.A.S., 71, 143.
Schweizer, F. 1980, Ap. J., 237, 303.

Smith, H. E., Burbidge, M., and Spinrad, H. 1976, Ap. J., 210, 627.

Smith, H. E., Junkkarinen, V. T., Spinrad, H., Grueff, G., and Vigotti, M. 1979, Ap. J., 231, 307.

Spinrad, H., Kron, R. G., and Hunstead, R. W. 1979, Ap. J. Suppl., 41, 701.

Spinrad, H., Liebert, J., Smith, H. E., and Hunstead, R. 1976, Ap. J. (Letters), 206, L79.

Spinrad, H., Stauffer, J., and Butcher, H. 1981, Ap. J., 244, 382

Spinrad, H., Stauffer, J., and Harlan, E. 1979, Pub. A.S.P., 91, 619.

Strom, K. M., and Strom, S. E. 1978, A.J., 83, 1293.

Strom, S. E., and Strom, K. M. 1978, A.J., 83, 732.

Strom, S. E., and Strom, K. M. 1979, A.J., 84, 1091

Tinsley, B. M. 1976, Ap. J. (Letters), 210, L49. 1978, Nature, 273, 208.

Tinsley, B. M., and Gunn, J. E. 1976, Ap. J., 203, 52.

Tinsley, B. M., and Larson, R. B. 1979, M.N.R.A.S., 186, 503.

S. DJorgovski and H. SpINRAD: Astronomy Department, University of California, Berkeley, CA 94720 